\title{
CATÉTER PERIDURAL LUMBAR Y TORÁCICO PARA ANALGESIA POSTOPERATORIA EN PEDIATRIA: EFECTIVIDAD ANALGESICA E INCIDENTES ASOCIADOS
}

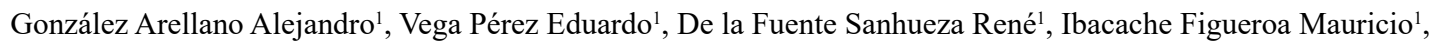
Rolle Pérez Augusto ${ }^{1}$, Fierro Neudorfer Claudio ${ }^{2}$

1 División de Anestesia, Pontificia Universidad Católica de Chile.

Servicio de Anestesia, Clínica Santa María.

Introducción: La analgesia peridural postoperatoria en pediatría es segura y efectiva. Sin embargo, el uso de catéteres lumbares para cirugía supraumbilical se relacionaría con analgesia insatisfactoria, en reportes que utilizan pocos catéteres torácicos. En teoría, la analgesia sería satisfactoria con catéteres peridurales insertados en relación al sitio de la incisión quirúrgica.

Objetivo General: Comparar la efectividad analgésica y la incidencia y tipo de incidentes asociados de catéteres peridurales lumbares y torácicos para analgesia postoperatoria en pacientes pediátricos.

Material y Métodos: Estudio de cohorte no concurrente, aprobado por ética. Utilizando la base de datos de la Unidad de Dolor del Servicio de Anestesia UC, se analizaron los pacientes de 0 a 18 años que recibieron analgesia peridural postoperatoria continua, entre octubre de 2011 y mayo de 2016. De acuerdo al sitio de inserción del catéter peridural, se dividió a los pacientes en grupo lumbar y grupo torácico; se registró información relacionada a: - Variables demográficas: edad; genero; clasificación ASA - Cirugía realizada. - Catéter peridural: nivel de inserción y duración in situ - Analgesia peridural: drogas, dosis, modalidad (infusión y/o bolos) y duración de utilización del catéter. - Analgésicos adicionales o de rescate - Dolor postoperatorio con escala CHIPPS o EVA según corresponda. - Número y tipo de incidentes asociados con el catéter o con las drogas utilizadas. Análisis estadístico: descriptivo; test student y/o test de Mann-Whitney; significativa $p<0,05$.

Resultados: Analizamos 222 pacientes: 123 con catéter lumbar y 99 con catéter torácico; variables demográficas en tabla 1. Todos los catéteres peridurales fueron instalados según el sitio de la cirugía: lumbares L1 a L4, torácicos T8 a T12. Las cirugías más frecuentes fueron: urológica, traumatológica y abdominal en el grupo lumbar; urológica, abdominal y de tórax en el grupo torácico. Hubo 22 incidentes en el grupo lumbar y 13 en el grupo torácico; principalmente náuseas y vómitos postoperatorios, retiro accidental u oclusión del catéter. No hubo diferencias entre los grupos $(\mathrm{p}=0,81)$. Los pacientes con catéter torácico tuvieron mayor dolor (EVA: $2(0-10) v s$ $0(0-10) ; \mathrm{p}<0,004)$, la duración del catéter y de la analgesia fue mayor $(2,96+1,06$ días $v s 2,53$ $+1,09$ días; $p=0,005$ ) que en aquellos con catéter lumbar. La solución analgésica peridural y

Tabla 1. Variables demográficas

\begin{tabular}{|llll|}
\hline & Catéter lumbar & Catéter torácico & Valor $\mathrm{p}$ \\
Edad (años) & $5,5+4,8$ & $6,9+5,4$ & 0.041 \\
Género (\%) & & & $\mathrm{NS}$ \\
Hombres & 56,2 & 66 & \\
Mujeres & 43,8 & 34 & \\
ASA: (\%) & & & $\mathrm{NS}$ \\
I & 46,3 & 48,5 & \\
II & 48 & 41,4 & 10,1 \\
III & 5,7 & & \\
\hline
\end{tabular}


los requerimientos analgésicos fueron similares entre ambos grupos $(p=0,46)$.

Conclusiones: En nuestros pacientes, la analgesia peridural postoperatoria produjo un adecuado alivio del dolor y se asoció a incidentes menores, resultados que son similares a los reportados en la literatura. Utilizamos frecuentemente catéteres peridurales insertados a nivel torácico en pacientes pediátricos, con efectividad analgésica y número de incidentes comparables a aquellos de los catéteres lumbares. Si bien, desde el punto de vista estadístico, hubo diferencias en la intensidad del dolor postoperatorio (catéteres torácicos $>$ catéteres lumbares), el bajo nivel de intensidad medido (CHIPPS o EVA) hace que esta diferencia sea clinicamente no relevante. Por tanto, la utilización de catéteres peridurales insertados en relación al sitio de la incisión quirúrgica produciría analgesia postoperatoria efectiva en pacientes pediátricos. 